\title{
Quantum fluctuations of a Bose-Josephson junction in a quasi-one-dimensional ring trap
}

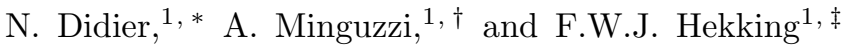 \\ ${ }^{1}$ Université Joseph Fourier, Laboratoire de Physique et de Modélisation \\ des Milieux Condensés, C.N.R.S. B.P. 166, 38042 Grenoble, France
}

\begin{abstract}
Using a Luttinger-liquid approach we study the quantum fluctuations of a Bose-Josephson junction, consisting of a Bose gas confined to a quasi one-dimensional ring trap which contains a localized repulsive potential barrier. For an infinite barrier we study the one-particle and two-particle static correlation functions. For the one-body density-matrix we obtain different power-law decays depending on the location of the probe points with respect to the position of the barrier. This quasi-long range order can be experimentally probed in principle using an interference measurement. The corresponding momentum distribution at small momenta is also shown to be affected by the presence of the barrier and to display the universal power-law behavior expected for an interacting 1D fluid. We also evaluate the particle density profile, and by comparing with the exact results in the Tonks-Girardeau limit we fix the nonuniversal parameters of the Luttinger-liquid theory. Once the parameters are determined from one-body properties, we evaluate the density-density correlation function, finding a remarkable agreement between the Luttinger liquid predictions and the exact result in the Tonks-Girardeau limit, even at the length scale of the Friedel-like oscillations which characterize the behavior of the density-density correlation function at intermediate distance. Finally, for a large but finite barrier we use the one-body correlation function to estimate the effect of quantum fluctuations on the renormalization of the barrier height, finding a reduction of the effective Josephson coupling energy, which depends on the length of the ring and on the interaction strength.
\end{abstract}

PACS numbers: 05.30.Jp, 67.85.-d

\section{INTRODUCTION}

The possibility to study Bose-Einstein condensates confined to ring traps constitutes one of the frontiers of the experimental progress with ultracold atomic gases [1]. The nontrivial topology of these traps together with the uniformity of the potential along the ring circumference makes them an ideal system for investigating persistent currents and superfluid properties of the gas.

While current experimental setups display relatively weak transverse (i.e. radial) confinements, we analyze in this work the case where the transverse confinement is so strong that only longitudinal (i.e. tangential) quasi$1 \mathrm{D}$ motion is allowed along the ring. In a quasi-1D geometry, the phase coherence properties of the gas are drastically changed with respect to their $3 \mathrm{D}$ counterparts. Phase fluctuations destroy true long-range order, and by increasing the interaction strength the gas changes from a quasi-condensate, i.e. a condensate with fluctuating phase [2, 3] to a Tonks-Girardeau gas [4], where repulsions are so strong that they mimic the effect of Pauli pressure in a Fermi gas and the condensate is strongly depleted. Here we consider such a onedimensional ring trap containing a localized repulsive potential which creates a "weak link" connecting the two

\footnotetext{
*Electronic address: Nicolas.Didier@grenoble.cnrs.fr

${ }^{\dagger}$ Electronic address: Anna.Minguzzi@grenoble.cnrs.fr

‡Electronic address: Frank.Hekking@grenoble.cnrs.fr
}

ends of the loop (see Fig. 1), a situation that may be viewed as a realization of a Bose-Josephson junction. Bose-Josephson junctions have been already experimentally realized using a double-well geometry and arrays [5]. In the configuration considered here, quantum fluctuations tend to destroy the phase coherence along the ring, while the tunneling of bosons between the ends of the loop favors a well-defined phase difference across the barrier. We will study the interplay between these competing effects.

We start by investigating how the presence of the barrier affects the quantum fluctuations and hence the coherence properties of a Bose-Josephson junction in a quasi$1 \mathrm{D}$ ring at arbitrary values of the interaction strength. In the absence of the barrier, these properties have been extensively studied, employing a variety of techniques, from low-energy Luttinger-liquid approaches and conformal field theory [6, 7], to exact methods in the TonksGirardeau regime [8, 9]. In the presence of an infinitely high barrier, using the Luttinger liquid approach for a finite ring, we evaluate here the first-order correlation function, which describes the decay of phase coherence along the ring, and recover a previous result from conformal field theory [6]. We then compare the results for the one-body density matrix and for the particledensity profile along the ring with the corresponding exact results in the Tonks-Girardeau limit of impenetrable bosons obtained through a Bose-Fermi mapping method [4]. This enables us to determine the numerical values of the nonuniversal parameters of the Luttingerliquid theory. Knowing these parameters we estimate 


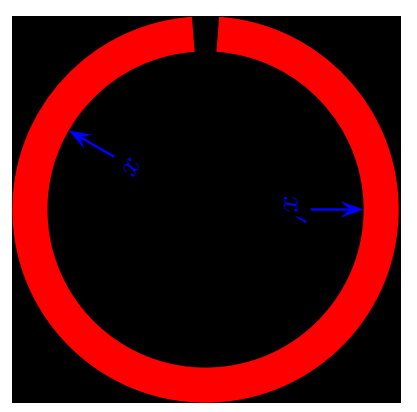

FIG. 1: Sketch of the Bose-Josephson junction on a ring trap studied in this work.

two-particle properties such as the density-density correlation function. Finally, we turn to the case of a large but finite barrier, treating the tunneling across the barrier as a perturbation. We use the results for the first-order correlation function in the infinite-barrier limit to estimate the effect of the quantum fluctuations on the effective height of the barrier, i.e. on the Josephson coupling energy. As a result, we predict how the renormalization of the Josephson energy depends on the ring length and on the interaction strength.

\section{LUTTINGER LIQUID DESCRIPTION FOR A RING WITH A LOCALIZED BARRIER}

We consider $N$ bosons confined to a uniform, quasi-1D ring-shaped trap of circumference $L$ to which a localized repulsive potential $V_{\text {barr }}(x)$, located at $x=0 \equiv L$, has been superimposed. The bosons interact with each other through a repulsive contact potential $v\left(x-x^{\prime}\right)=$ $g \delta\left(x-x^{\prime}\right)$. The corresponding Hamiltonian in terms of the bosonic field operators $\Psi(x), \Psi^{\dagger}(x)$ reads

$$
\begin{aligned}
\mathcal{H}= & \int \mathrm{d} x \Psi^{\dagger}(x)\left(\frac{\hbar^{2}}{2 m} \nabla^{2}+V_{\mathrm{barr}}(x)\right) \Psi(x) \\
& +\frac{g}{2} \int \mathrm{d} x \Psi^{\dagger}(x) \Psi^{\dagger}(x) \Psi(x) \Psi(x) .
\end{aligned}
$$

The presence on the ring of the (very large) barrier potential will be taken into account below by imposing open boundary conditions at $x=0=L$ (see Sec. IB ) and by adding a tunnel term (see Sec. VI).

\section{A. Low-energy theory}

In order to evaluate the equilibrium correlation functions at large and intermediate distances we adopt the Luttinger liquid approach, i.e. we approximate the system Hamiltonian (11) by the following effective lowenergy Hamiltonian in terms of the fields $\theta(x)$ and $\phi(x)$ which describe the density and phase fluctuations on the ring [10],

$$
\mathcal{H}_{L L}=\frac{\hbar v_{s}}{2 \pi} \int_{0}^{L} \mathrm{~d} x\left[K(\nabla \phi(x))^{2}+\frac{1}{K}(\nabla \theta(x))^{2}\right]
$$

The parameters $K$ and $v_{s}$ are related to the microscopic interaction parameter of the original Hamiltonian (10) [6], the phase field $\phi(x)$ is related to the velocity of the fluid $v(x)=\hbar \nabla \phi(x) / m$ and the field $\theta(x)$ defines the fluctuations in the density profile $\rho(x)$ according to

$$
\rho(x)=\left[\rho_{0}+\Pi(x)\right] \sum_{m=-\infty}^{+\infty} \mathrm{e}^{2 m i \theta(x)+i 2 \pi m \rho_{0} x+2 i m \theta_{B}}
$$

where $\rho_{0}=N / L$ is the average density of the fluid, $\Pi(x)=\nabla \theta(x) / \pi$ and $\theta_{B}$ is a constant fixing the position of the first particle with respect to the origin of the $x$ axis. The fields $\Pi$ and $\phi$ satisfy the commutation relation [6, 10]

$$
\left[\Pi(x), \phi\left(x^{\prime}\right)\right]=i \delta\left(x-x^{\prime}\right)
$$

Note that this approach includes not only the lowestorder hydrodynamic expression for the density fluctuation at long wavelength: the higher order terms in the sum (3) enable the description of the discrete nature of the particles up to distances $\alpha \sim 1 / \rho_{0}$. Our approach does not allow however to probe shorter distance scales because of the assumption of linear phonon dispersion modes in the effective Hamiltonian (2). The bosonic field operator is obtained from the hydrodynamic expression $\Psi^{\dagger}(x)=\sqrt{\rho(x)} e^{-i \phi(x)}$ and reads

$$
\begin{aligned}
\Psi^{\dagger}(x) & =\mathcal{A} \sqrt{\rho_{0}+\Pi(x)} \\
& \times \sum_{m=-\infty}^{+\infty} \mathrm{e}^{2 m i \theta(x)+2 i m \pi \rho_{0} x+2 i m \theta_{B}} \mathrm{e}^{-i \phi(x)},
\end{aligned}
$$

where $\mathcal{A}$ is a nonuniversal constant, the value of which depends on the way the Luttinger liquid approach is regularized in the short wavelength limit. This issue will be discussed in Sec. VA below, where the value of the constants $\mathcal{A}$ and $\theta_{B}$ will be fixed. The field operators $\Psi$ and $\Psi^{\dagger}$ as defined through Eq. (5) satisfy the standard bosonic commutation relations as a consequence of the commutation relation (4) among the field operators $\Pi$ and $\phi$.

\section{B. Mode expansion of the Luttinger fields $\theta$ and $\phi$ with open boundary conditions}

We start by considering the case of an infinitely high barrier, which corresponds to a ring with open boundary conditions. In Sec. VI we will treat the case of a large, finite barrier by considering the tunneling among the two sides of the barrier as a perturbation. 
In order to evaluate the first- and second-order correlation functions for the bosons on the ring junction, we derive here the expansion of the fields $\Pi(x)=\nabla \theta(x) / \pi$ and $\phi(x)$ in terms of canonical bosonic annihilation and creation operators $b_{k}$ and $b_{k}^{\dagger}$ satisfying the commutation relations $\left[b_{k}, b_{k^{\prime}}^{\dagger}\right]=\delta_{k, k^{\prime}}$. Specifically, we expand the operators $\phi(x)$ and $\Pi(x)$ in Fourier modes for $x \in[0, L]$ :

$$
\begin{aligned}
\phi(x) & =\sum_{j=-\infty}^{+\infty}\left[\phi_{1, j} b_{k_{j}}+\phi_{2, j} b_{k_{j}}^{\dagger}\right] \mathrm{e}^{i k_{j} x}, \\
\Pi(x) & =\sum_{j=-\infty}^{+\infty}\left[\Pi_{1, j} b_{k_{j}}+\Pi_{2, j} b_{k_{j}}^{\dagger}\right] \mathrm{e}^{i k_{j} x}
\end{aligned}
$$

where we have set $k_{j}=\frac{2 \pi}{L} p j$. The constant $p$ and the complex coefficients $\phi_{1, j}, \phi_{2, j}, \Pi_{1, j}$ and $\Pi_{2, j}$ are determined by imposing three constraints: (i) the commutations rules $\left[\Pi(x), \phi\left(x^{\prime}\right)\right]=i \delta\left(x-x^{\prime}\right)$; (ii) the open boundary conditions, which imply vanishing current density i.e. $\nabla \phi(0)=\nabla \phi(L)=0, \nabla \Pi(0)=\nabla \Pi(L)=0$; (iii) reduction of the Hamiltonian to the diagonal form $\mathcal{H}=\sum_{k_{j}} \hbar \omega_{k_{j}}\left[b_{k_{j}}^{\dagger} b_{k_{j}}+\frac{1}{2}\right]$. In order to take into account that we are using an approximate, long wavelength theory, we introduce a short distance cutoff $\alpha \sim \rho_{0}{ }^{-1}$ in the sum over the modes. The final result reads

$$
\begin{aligned}
\phi(x) & =\phi_{0} \\
& +\frac{1}{\sqrt{K}} \sum_{j=1}^{\infty} \frac{1}{\sqrt{j}} \cos (\pi j x / L) \mathrm{e}^{-\frac{\pi j \alpha}{2 L}}\left[b_{k_{j}}+b_{k_{j}}^{\dagger}\right],(8) \\
\Pi(x) & =\Pi_{0} \\
& +i \frac{\sqrt{K}}{L} \sum_{j=1}^{\infty} \sqrt{j} \cos (\pi j x / L) \mathrm{e}^{-\frac{\pi j \alpha}{2 L}}\left[b_{k_{j}}-b_{k_{j}}^{\dagger}\right](9)
\end{aligned}
$$

the latter implying

$$
\begin{aligned}
\theta(x) & =\pi \Pi_{0} x \\
& +i \sqrt{K} \sum_{j=1}^{\infty} \frac{1}{\sqrt{j}} \sin (\pi j x / L) \mathrm{e}^{-\frac{\pi j \alpha}{2 L}}\left[b_{k_{j}}-b_{k_{j}}^{\dagger}\right](, 10)
\end{aligned}
$$

where $k_{j}=\pi j / L(p=1 / 2)$. The zero mode $\Pi_{0}$ is directly related to the particle number operator through normalization: $\Pi_{0}=\left(N-N_{0}\right) / L$ where $N_{0}=\langle N\rangle=\rho_{0} L$. It is conjugate to the zero-mode phase operator $\phi_{0}$ such that $\left[\Pi_{0}, \phi_{0}\right]=i$. Using this fact, one can explicitly check the commutation rule between $\theta(x)$ and $\phi\left(x^{\prime}\right)$ from the mode expansions (8) and (10); it turns out to be $\left[\theta(x), \phi\left(x^{\prime}\right)\right]=i \pi \mathrm{u}\left(x-x^{\prime}\right)$, where $\mathrm{u}$ is the unit step function, consistent with (4). Finally, inspection of the diagonalized form of the Hamiltonian yields the linear dispersion relation $\omega_{k_{j}}=\hbar v_{S} k_{j}$ for the modes.

\section{EXACT DESCRIPTION IN THE TONKS-GIRARDEAU LIMIT $\mathrm{K}=1$}

In the limit of infinitely strong repulsion between the bosons, which corresponds to the value $K=1$ for the Luttinger liquid parameter, an exact solution exists for the bosonic many-body wavefunction $\Phi\left(x_{1}, \ldots x_{N}\right)$ (in first quantization). We shall use it throughout this paper in order to test the results of the Luttinger-liquid theory in the limit $K=1$, thereby fixing the values of its nonuniversal parameters.

The solution, due to Girardeau [4], is obtained by mapping the bosons onto a gas of noninteracting, spinpolarized fermions subject to the same external potential. The bosonic many-body wavefunction $\Phi\left(x_{1}, \ldots x_{N}\right)$ is then obtained in terms of the fermionic one as

$$
\Phi\left(x_{1}, \ldots x_{N}\right)=A\left(x_{1}, \ldots x_{N}\right) \Phi_{F}\left(x_{1} \ldots x_{N}\right),
$$

where the mapping function $A\left(x_{1}, \ldots x_{N}\right)=$ $\Pi_{1 \leq j \leq \ell \leq N} \operatorname{sign}\left(x_{j}-x_{\ell}\right)$ ensures the proper symmetry under exchange of two bosons. The fermionic wavefunction is given by $\Phi_{F}\left(x_{1}, \ldots x_{N}\right)=(1 / \sqrt{N !}) \operatorname{det}\left[\psi_{j}\left(x_{k}\right)\right]_{j, k=1 \ldots N}$, $\psi_{j}(x)$ being the single particle orbitals for the given external potential. Note that $\Phi_{F}$ vanishes every time two particles meet as required by Pauli's principle, and hence describes well the impenetrability condition $g \rightarrow \infty$ for the bosons. In our specific case, the orbitals for a ring of circumference $L$ and open boundary conditions are

$$
\psi_{j}(x)=\sqrt{(2 / L)} \sin (\pi j x / L)
$$

with $j=1, \ldots, \infty$.

As a consequence of the Bose-Fermi mapping, all the bosonic properties which do not depend on the sign of the many-body wavefunction coincide with the corresponding ones of the mapped Fermi gas. This is the case e.g. for the particle density profile and for the densitydensity correlation function. Other properties like the one-body density matrix and the momentum distribution are instead markedly different for bosons as compared to fermions. In particular, the calculation of the one-body density matrix requires in principle the calculation of a (N-1)-dimensional integral, which is known to simplify in some cases. Examples are the homogeneous gas with periodic boundary conditions [8] or the case of a harmonic confinement [9].

\section{ONE-BODY DENSITY MATRIX AND MOMENTUM DISTRIBUTION IN THE INFINITE-BARRIER LIMIT}

In this section we focus on the one-body density matrix and on the momentum distribution for the case of a bosonic ring of circumference $L$ with an infinite barrier, with the aim of analyzing the differences with respect to the case of an infinite system, as well as to the case of a ring in the absence of the barrier. 


\section{A. Contribution from phase fluctuations to the one-body density matrix}

The one-body density matrix, defined as $\mathcal{G}\left(x, x^{\prime}\right)=$ $\left\langle\Psi^{\dagger}(x) \Psi\left(x^{\prime}\right)\right\rangle$ yields a measure of the coherence along the ring. It is possible to measure the one-body density matrix and off-diagonal long range order experimentally by measuring the interference pattern of atomic matter waves coming from two holes in the trap (see e.g. 11] for the case of a cigar-shaped 3D Bose gas). According to Eq. (5) the bosonic field operator has three contributions: (i) the phase $\phi(x)$ (ii) the density fluctuation $\Pi(x)$, and (iii) the higher harmonics of order $2 m \theta(x)$ of the density. The most important contribution to the one-body density matrix at large distances is the one due to the phase fluctuations which correspond to the lowest-energy modes of the bosonic fluid in the ring (see e.g. [2, 7]), while the two latter contributions give rise to subleading corrections which we do not analyze further here.

To lowest order we approximate the bosonic field operator (5) as $\Psi(x) \simeq \mathcal{A} \sqrt{\rho_{0}} e^{i \phi(x)}$; the problem then reduces to the computation of the quantum average $\mathcal{G}_{0}\left(x, x^{\prime}\right)=\mathcal{A}^{2} \rho_{0}\left\langle e^{-i \phi(x)} e^{i \phi\left(x^{\prime}\right)}\right\rangle$. Since the Luttinger-liquid Hamiltonian (2) is quadratic in the field $\phi(x)$ we immediately obtain $\mathcal{G}_{0}\left(x, x^{\prime}\right)=$ $\mathcal{A}^{2} \rho_{0} \exp \left(-\frac{1}{2}\left\langle\left[\phi(x)-\phi\left(x^{\prime}\right)\right]^{2}\right\rangle\right)$. The phase-phase correlation function is evaluated with the help of the mode expansion (8); using the fact that the groundstate average over the bosonic modes is $\left\langle\left(b_{k}+b_{k}^{\dagger}\right)\left(b_{l}+\right.\right.$ $\left.\left.b_{l}^{\dagger}\right)\right\rangle=\delta_{k l}$ and the property $\sum_{j=1}^{\infty} \frac{1}{j} \mathrm{e}^{-\alpha j} \cos (\gamma j)=$ $-\frac{1}{2} \ln \left[1-2 \cos \gamma \mathrm{e}^{-\alpha}+\mathrm{e}^{-2 \alpha}\right]$ one readily obtains

$$
\begin{aligned}
\left\langle\phi(x) \phi\left(x^{\prime}\right)\right\rangle=-\frac{1}{4 K} \ln [ & (\pi / L)^{4}\left(\alpha^{2}+d^{2}\left(x-x^{\prime} \mid 2 L\right)\right) \\
& \left.\times\left(\alpha^{2}+d^{2}\left(x+x^{\prime} \mid 2 L\right)\right)\right]
\end{aligned}
$$

$d(x \mid L)=L|\sin (\pi x / L)| / \pi$ being the cord function. This leads to

$$
\begin{aligned}
& \mathcal{G}_{0}\left(x, x^{\prime}\right)=\rho_{0} b_{0,0} \\
\times & {\left[\frac{\rho_{0}^{-2} \sqrt{\left[\alpha^{2}+d^{2}(2 x \mid 2 L)\right]\left[\alpha^{2}+d^{2}\left(2 x^{\prime} \mid 2 L\right)\right]}}{\left[\alpha^{2}+d^{2}\left(x-x^{\prime} \mid 2 L\right)\right]\left[\alpha^{2}+d^{2}\left(x+x^{\prime} \mid 2 L\right)\right]}\right]^{\frac{1}{4 K}} }
\end{aligned}
$$

where we have introduced the nonuniversal constant $b_{0,0}=|\mathcal{A}|^{2}\left(\rho_{0} \alpha\right)^{\frac{1}{2 K}}$. The above expression (14) yields the leading-order term for the one-body density matrix at large distances. By taking the limit $\alpha \rightarrow 0$ we recover the result obtained in [6] using the methods of conformal field theory.

If the distance among $x$ and $x^{\prime}$ is large compared to the cutoff length $\alpha$, the one-body density matrix displays a power-law decay of the form $\mathcal{G}_{0}\left(x, x^{\prime}\right) \propto\left|x-x^{\prime}\right|^{-\gamma}$, where the exponent $\gamma$ can be derived from the expression (14), and in particular depends on the location of the probed points 6 . Indeed, if the two points are away from the edges one finds $\gamma=\frac{1}{2 K}$, which corresponds to the result obtained in the thermodynamic limit [10] whereas if they approach the edges (i.e. $x \lesssim \alpha$ and $L-x^{\prime} \lesssim \alpha$ ) the exponent is $\gamma=\frac{1}{K}$, a result known in the context of quantum phase fluctuations in a 1D superconducting wire of length $L$ [12]. In the case where one point is at one edge and the other in the bulk we obtain $\gamma=\frac{3}{4 K}$. These three different behaviors are illustrated in Fig. 2, where we plot the one-body density matrix $\mathcal{G}_{0}\left(x, x^{\prime}\right)$ as a function of $x^{\prime}$ for various choices of the probe point $x$. In the same figure we display also the behavior for a homogeneous ring in absence of the barrier, obtained by a procedure analogous to the one outlined above,

$$
\mathcal{G}_{0}^{\text {hom }}\left(x, x^{\prime}\right)=\rho_{0} b_{0,0}\left[\frac{\rho_{0}^{-2}}{\alpha^{2}+d^{2}\left(x-x^{\prime} \mid L\right)}\right]^{\frac{1}{4 K}} .
$$

Note that, as the coordinate $x^{\prime}$ runs along the ring, in the presence of the barrier the coherence decreases monotonically, while if the barrier is absent coherence is recovered as $x^{\prime}$ approaches $L-x$.

The different power-law behaviors are in principle observable for a quasi-1D Bose gas in a ring trap geometry; it is required to have a high barrier well localized on a length scale $\alpha$.

\section{B. Momentum distribution}

We proceed by studying the momentum distribution $n(q)$, obtained by Fourier transformation of the one-body density matrix with respect to the relative variable,

$$
n(q)=\int_{0}^{L} \mathrm{~d} x \int_{0}^{L} \mathrm{~d} x^{\prime} \mathrm{e}^{-i q\left(x-x^{\prime}\right)} \mathcal{G}\left(x, x^{\prime}\right) .
$$

We have resorted to a numerical calculation for the evaluation of the momentum distribution taking as input the one-body density matrix obtained in Eq. (14). This allows to estimate the main features of the momentum distribution at wavevectors $q$ smaller than the cutoff wavevector $q_{c} \sim 1 / \alpha$. The behavior at large wavevectors $q \gg q_{c}$ needs an accurate treatment of the short-distance behavior of the many-body wavefunction [13, 14] and is beyond the regime of validity of the Luttinger-liquid method. The result for the momentum distribution is illustrated in Fig. 3 for two values of the boson number in the ring, and at varying interaction strength. As a general feature (see the inset of Fig. 31), we observe that at intermediate values of $q$ the momentum distribution displays a power-law behavior $n(q) \sim q^{1 /(2 K)-1}$ with the same power predicted for a homogeneous ring (see e.g. 7]). This result is readily understood as the different power laws described in Sec. IVA only occur at the edge of the integration region with a negligible weight with respect to the bulk contribution. Still, by comparing the details of the momentum distribution of the ring with the barrier with the momentum distribution of a uniform ring, (see the main panel of Fig. 3), we find that 


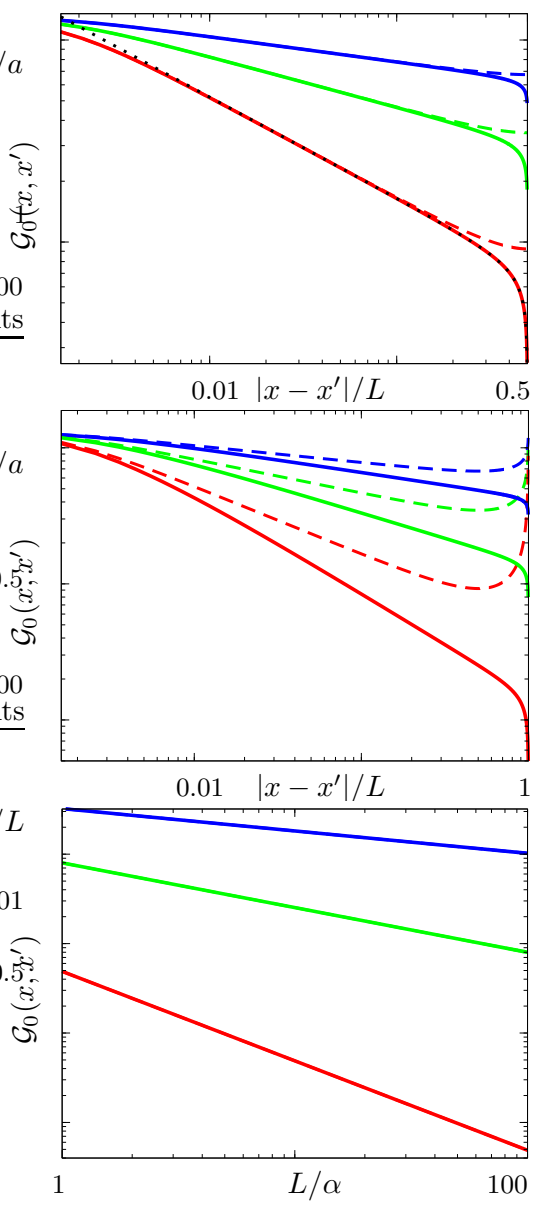

FIG. 2: (Color online) Top and middle panels: one-body density matrix in logarithmic scale (arbitrary units) as a function of the coordinate $x^{\prime}$ (in units of the ring circumference $L$ ) for various choices of the coordinate $x$ with respect to the position of the barrier, located at $x=0$ : top panel $x \simeq 0$, middle panel $x=L / 2$. Bottom panel: one-body density matrix in logarithmic scale (arbitrary units) taken at $x \simeq 0$ and $x^{\prime} \simeq L$ as a function of the length $L$ of the ring (in units of the average interparticle distance $\alpha$ ). In each panel we plot three values of the parameter $K$ (from bottom to top $K=1, K=2$ and $K=4)$; the solid lines correspond to the results from Eq. (14) and the dashed lines are the solution (15) for a homogeneous ring (periodic boundary conditions). The dotted line in the top panel is the exact solution for a Tonks-Girardeau gas in the thermodynamic limit. The linear behavior of $\mathcal{G}_{0}$ in logarithmic scale correspond to the predicted power law decays with various exponents $\gamma$ (top panel, $\gamma=1 / 2 K$, middle panel $\gamma=3 / 4 K$ and bottom panel $\gamma=1 / K)$.

in the presence of the barrier the momentum distribution is decreased at small momenta. This is in agreement with the fact that the barrier reduces the coherence along the ring. The result is reminiscent of the one obtained for a 1D gas in presence of disorder [15], where the reduction of the momentum distribution at small momenta is also observed.

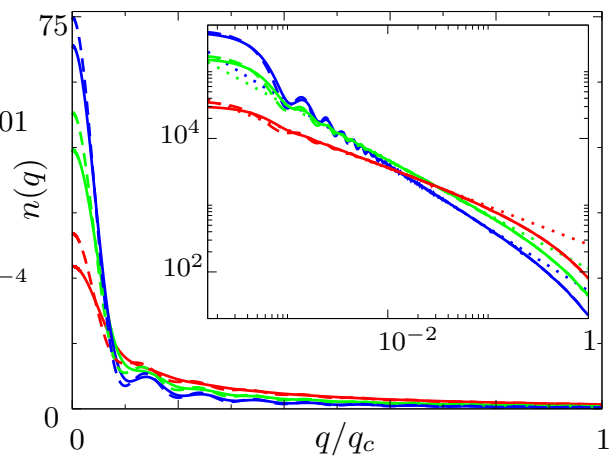

FIG. 3: (Color online) Momentum distribution $n(q)$ in units of $2 \pi|\mathcal{A}|^{2} \alpha$ as a function of the wavevector $q$ in units of the cutoff momentum $q_{c}=1 / \alpha$ for $N=10$ bosons on a ring with an infinite barrier (solid lines) and for a homogeneous ring in absence of the barrier (dashed lines) for various values of the Luttinger parameter $K$ (from top to bottom $K=4,2,1$ ). The inset shows the same quantity (in logarithmic scale, arbitrary units, same line conventions as the main figure) evaluated for $N=10^{3}$ bosons. The dotted lines indicate the predicted power law decays $q^{1 /(2 K)-1}$.

\section{PARTICLE-DENSITY PROFILE AND DENSITY-DENSITY CORRELATION FUNCTION IN THE INFINITE-BARRIER LIMIT}

Extending the quantum average techniques outlined in Sec. IV to the limit of an infinitely high barrier it is possible to evaluate also the inhomogeneous particle-density profile and the density-density correlation functions. Interference between particles incident on and reflected by the barrier leads to the occurrence of Friedel-like oscillations in the density profile and in its correlator, which are typical of strongly correlated 1D fluids. We describe here these oscillations within the Luttinger liquid approach, for any value of the coupling strength, finding that they are more and more marked as the coupling strength increases. In the Tonks-Girardeau limit of infinite boson-boson repulsion we compare the predictions of the Luttinger-liquid approach with the exact results, which enables us to fix the nonuniversal parameters of the latter.

\section{A. Friedel oscillations in the particle density profile}

We compute the particle-density profile by taking the quantum average $\langle\rho(x)\rangle$ of the density operator (3) on the ground state, namely

$$
\langle\rho(x)\rangle / \rho_{0}=\sum_{m=-\infty}^{+\infty}\left\langle\left(1+\Pi(x) / \rho_{0}\right) \mathrm{e}^{2 m i \theta(x)}\right\rangle \mathrm{e}^{i 2 \pi m \rho_{0} x+2 i m \theta_{B}} .
$$

To evaluate the quantum averages we exploit the fact that the Hamiltonian (2) is quadratic in the field $\theta(x)$ (and recall that $\Pi(x)=\nabla \theta(x) / \pi)$. We use the property 
that if $X$ and $Z$ are Gaussian variables then $\left\langle X \mathrm{e}^{Z}\right\rangle=$ $\langle X Z\rangle \mathrm{e}^{\frac{1}{2}\left\langle Z^{2}\right\rangle}$. Hence, we are left with the evaluation of the $\left\langle\theta(x) \theta\left(x^{\prime}\right)\right\rangle$ correlation function, to be taken at equal points $x=x^{\prime}$; the $\left\langle\Pi(x) \theta\left(x^{\prime}\right)\right\rangle$ correlation function is obtained from the previous one by taking the derivative with respect to the variable $x$. Using the mode expansion (10) and a procedure similar to the one outlined in Sec. IV we obtain

$$
\begin{aligned}
\left\langle\theta(x) \theta\left(x^{\prime}\right)\right\rangle & =\pi^{2}\left\langle\Pi_{0}^{2}\right\rangle x x^{\prime} \\
& -q \frac{K}{4} \ln \left[\frac{\alpha^{2}+d^{2}\left(x-x^{\prime} \mid 2 L\right)}{\alpha^{2}+d^{2}\left(x+x^{\prime} \mid 2 L\right)}\right],
\end{aligned}
$$

and

$$
\begin{aligned}
\left\langle\Pi(x) \theta\left(x^{\prime}\right)\right\rangle & =\pi\left\langle\Pi_{0}^{2}\right\rangle x^{\prime}+\frac{K}{2 \pi} \frac{d\left(x+x^{\prime} \mid L\right)}{\alpha^{2}+d^{2}\left(x+x^{\prime} \mid 2 L\right)} \\
& -\frac{K}{2 \pi} \frac{d\left(x-x^{\prime} \mid L\right)}{\alpha^{2}+d^{2}\left(x-x^{\prime} \mid 2 L\right)} \operatorname{sign}\left(x-x^{\prime}\right) .
\end{aligned}
$$

The final expression for the density profile reads

$$
\begin{aligned}
\frac{\langle\rho(x)\rangle}{\rho_{0}} & =1+2 \sum_{m=1}^{\infty}\left(\frac{\alpha}{d(2 x \mid 2 L)}\right)^{m^{2} K}\left[\cos \left(2 m \pi \rho_{0} x+2 m \theta_{B}\right)\right. \\
& \left.-\frac{m K}{\pi \rho_{0}} \sin \left(2 m \pi \rho_{0} x+2 m \theta_{B}\right) \frac{d(2 x \mid L)}{d^{2}(2 x \mid 2 L)}\right] .
\end{aligned}
$$

The density profile is modulated by oscillations with wavevector multiples of $2 \pi \rho_{0}$. Notice that in the case $K=1$, where the system can be mapped onto a noninteracting spin-polarized Fermi gas, the wavevectors of the oscillation are multiples of $2 k_{F}$, where $k_{F}=\pi \rho_{0}$ is the Fermi wavevector, and hence correspond to the wellknown Friedel oscillations [16]. For the case of generic $K$ the $m=1$ oscillations decay with the power law $x^{-K}$ (see e.g. [17]).

Let us now concentrate on the case $K=1$. In the thermodynamic limit $\left(L \rightarrow \infty, N \rightarrow \infty\right.$, at fixed $\rho_{0}=$ $N / L$ ) the expression (20) for the density profile at short distances (to $O(1 / x)$ ) reduces to

$$
\frac{\langle\rho(x)\rangle}{\rho_{0}} \simeq 1+\frac{\alpha \cos \left(2 \pi \rho_{0} x+2 \theta_{B}\right)}{x} .
$$

This can be compared with the thermodynamic limit of the exact expression derived using the Bose-Fermi mapping [9, 18]

$$
\frac{\langle\rho(x)\rangle}{\rho_{0}} \simeq 1-\frac{\sin \left(2 \pi \rho_{0} x\right)}{2 \pi \rho_{0} x},
$$

allowing us to fix the coefficients $\alpha$ and $\theta_{B}$ to the values $\alpha=1 /\left(2 \pi \rho_{0}\right)$ and $\theta_{B}=\pi / 4$. Note that the latter choice for $\theta_{B}$ is in agreement with the condition $\theta_{B} \neq 0, \pm \pi, \pm 2 \pi \ldots$ obtained by imposing that the particle density profile should vanish at $x=0$ and $x=L[\overline{6}]$. Once the constants $\alpha$ and $\theta_{B}$ are chosen, the constant $\mathcal{A}$ in Eq. (5) can be fixed by comparing the expression for

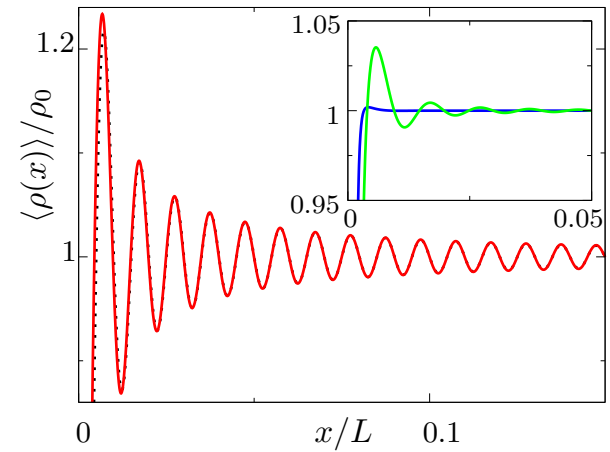

FIG. 4: (Color online) Particle density profile $\langle\rho(x)\rangle$ in units of the average particle density $\rho_{0}$ as a function of the spatial coordinate $x$ along the ring (in units of the ring circumference $L$ ) for various values of the parameter $K$. Main figure, $K=1$ (solid line: result from the Luttinger-liquid model, dotted line, exact result from the Bose-Fermi mapping); inset, $K=2$ (top green line) and $K=4$ (bottom blue line).

the coefficient $b_{00}$ entering Eq. (14) for the one-body density matrix with the exact value $b_{00}^{\text {exact }}=2^{-1 / 3} \sqrt{\pi e} A_{G}^{-6} \sim$ 0.521 [8, 9] where $A_{G}=1.282 \ldots$ is Glaisher's constant. The result is $|\mathcal{A}|^{2}=2^{1 / 6} \pi \mathrm{e}^{1 / 2} A_{G}^{-6} \sim 1.307$. This value has been used in plotting Fig. 2.

In Figure 4 we illustrate the density profiles for various values of $K$, obtained by the Luttinger-liquid expression (20) using the above choice for $\alpha$ and $\theta_{B}$ [19]. The figure displays also the exact result for the density profile obtained from the Bose-Fermi mapping, $\rho^{\text {exact }}(x)=$ $\sum_{j=1}^{N}\left|\psi_{j}(x)\right|^{2}$, where the single-particle orbitals $\psi_{j}(x)$ are defined in Eq. (12). The comparison shows how our choice of parameters $\alpha$ and $\theta_{B}$ reproduces extremely well the density profile oscillations even on a finite ring. The figure also illustrates how the Friedel oscillations display maximal amplitude in the strongly interacting limit $K=1$.

\section{B. Density-density correlation function from Luttinger-liquid theory}

We turn now to the density-density correlation function $S\left(x, x^{\prime}\right)=\left\langle\rho(x) \rho\left(x^{\prime}\right)\right\rangle-\langle\rho(x)\rangle\left\langle\rho\left(x^{\prime}\right)\right\rangle$. This quantity encodes the information on the structure of the fluid, i.e. on the correlations between density modulations at different parts of the fluid, while it vanishes for an ideal Bose gas. The Fourier transform of the density-density correlation function with respect to the relative variable is directly accessible experimentally by light-scattering methods (see e.g. [20] and references therein).

The density-density correlation function is obtained with the quantum average method described in Sec. IV 
and $\mathrm{V}$. One has to compute

$$
\begin{aligned}
\left\langle\rho(x) \rho\left(x^{\prime}\right)\right\rangle & =\sum_{m, m^{\prime}=-\infty}^{+\infty} \mathrm{e}^{2 i\left(m-m^{\prime}\right) \theta_{B}} \mathrm{e}^{i 2 \pi \rho_{0}\left(m x-m^{\prime} x^{\prime}\right)} \\
& \times\left\langle\left[\rho_{0}{ }^{2}+\rho_{0}\left(\Pi(x)+\Pi\left(x^{\prime}\right)\right)+\Pi(x) \Pi\left(x^{\prime}\right)\right]\right. \\
& \left.\times \mathrm{e}^{2 i\left(m \theta(x)-m^{\prime} \theta\left(x^{\prime}\right)\right)}\right\rangle .
\end{aligned}
$$

The average can be performed using the general result for Gaussian variables $\left\langle X Y \mathrm{e}^{Z}\right\rangle=$ $(\langle X Y\rangle+\langle X Z\rangle\langle Y Z\rangle) \mathrm{e}^{\frac{1}{2}\left\langle Z^{2}\right\rangle}$. The novel correlator needed for the calculation in addition to Eqs. (18) and (19) is

$$
\begin{aligned}
\left\langle\Pi(x) \Pi\left(x^{\prime}\right)\right\rangle & =-\frac{K}{2 \pi^{2}}\left[\frac{d^{2}\left(x-x^{\prime} \mid 2 L\right)-\alpha^{2} \cos \left(\pi\left(x-x^{\prime}\right) / L\right)}{\left(d^{2}\left(x-x^{\prime} \mid 2 L\right)+\alpha^{2}\right)^{2}}\right. \\
& \left.+\frac{d^{2}\left(x+x^{\prime} \mid 2 L\right)-\alpha^{2} \cos \left(\pi\left(x+x^{\prime}\right) / L\right)}{\left(d^{2}\left(x+x^{\prime} \mid 2 L\right)+\alpha^{2}\right)^{2}}\right] \\
& +\left\langle\Pi_{0}^{2}\right\rangle .
\end{aligned}
$$

The final result reads

$$
\begin{aligned}
\left\langle\rho(x) \rho\left(x^{\prime}\right)\right\rangle= & \sum_{m, m^{\prime}=-\infty}^{+\infty}\left(\rho_{0} \alpha\right)^{\left(m^{2}+m^{\prime 2}\right) K} \mathrm{e}^{2 i\left(m-m^{\prime}\right) \theta_{B}} \mathrm{e}^{i 2 \pi \rho_{0}\left(m x-m^{\prime} x^{\prime}\right)} \\
\times & {\left[\rho_{0}^{2}+\left\langle\Pi(x) \Pi\left(x^{\prime}\right)\right\rangle+2 i \rho_{0}\left\langle\left(\Pi(x)+\Pi\left(x^{\prime}\right)\right)\left(m \theta(x)-m^{\prime} \theta\left(x^{\prime}\right)\right)\right\rangle\right.} \\
& \left.\quad-4\left\langle\Pi(x)\left(m \theta(x)-m^{\prime} \theta\left(x^{\prime}\right)\right)\right\rangle\left\langle\Pi\left(x^{\prime}\right)\left(m \theta(x)-m^{\prime} \theta\left(x^{\prime}\right)\right)\right\rangle\right] \\
\times & \left(\frac{\alpha^{2}+d^{2}\left(x+x^{\prime} \mid 2 L\right)}{\alpha^{2}+d^{2}\left(x-x^{\prime} \mid 2 L\right)}\right)^{m m^{\prime} K}\left(\frac{\rho_{0}^{-2}}{\alpha^{2}+d^{2}(2 x \mid 2 L)}\right)^{\frac{1}{2} m^{2} K}\left(\frac{\rho_{0}^{-2}}{\alpha^{2}+d^{2}\left(2 x^{\prime} \mid 2 L\right)}\right)^{\frac{1}{2} m^{\prime 2} K} .
\end{aligned}
$$

This equation displays the general structure of the density-density correlations to all orders in $m$ and $m^{\prime}$, and by considering only the first terms of the expansion $m, m^{\prime}=0, \pm 1$ we recover the known results [6, 7].

We proceed by comparing the density-density correlation function $S\left(x, x^{\prime}\right)$ with the exact result for $K=1$. The latter is obtained from the Bose-Fermi mapping as [20]:

$$
S^{\text {exact }}\left(x, x^{\prime}\right)=-\left[\sum_{j=1}^{N} \psi_{j}{ }^{*}(x) \psi_{j}\left(x^{\prime}\right)\right]^{2},
$$

where the single-particle orbitals $\psi_{j}(x)$ are defined in Eq. (12).

Figure 5 displays the results obtained from the Luttinger-liquid method at various values of the Luttinger parameter $K$, using the choice of parameters $\alpha$ and $\theta_{B}$ determined from the density profile in Sec. VA and compares to the exact ones in the case $K=1$. The agreement found is very good, even for the Friedel-like oscillations at wave vector $k \sim 2 \pi \rho_{0}$; this is at the boundary of the expected regime of validity of the Luttingerliquid theory and illustrates how a reasonable choice of the non-universal parameters in the effective model allows for surprisingly accurate predictions.

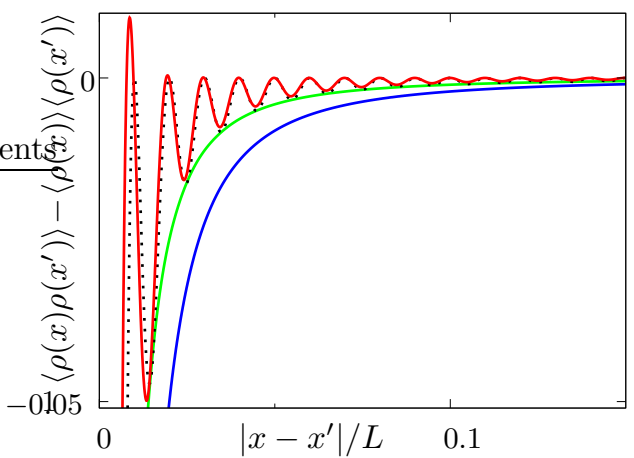

FIG. 5: (Color online) Density-density correlation function $S\left(x, x^{\prime}\right)$ (in units of $\rho_{0}^{2}$ ) from the Luttinger-liquid model as a function of the coordinate $x^{\prime}$ (in units of the ring circumference $L$ ), with $x=L / 2$ and for various values of the Luttinger parameter $K(K=1$, top red line, $K=2$, middle green line, $K=4$ bottom blue line). The dotted line corresponds to the exact solution obtained from the Bose-Fermi mapping in the case $K=1$.

\section{RENORMALIZATION OF JOSEPHSON ENERGY BY QUANTUM FLUCTUATIONS}

In this section we consider the effect of a finite barrier on the ring located at $x=0 \equiv L$, and described by a localized potential $V_{\text {barr }}(x)=\mathcal{T} \delta(x)$, where $\mathcal{T}$ is the tunneling strength of the barrier. This yields the following 
barrier term in the Hamiltonian (10):

$$
\mathcal{H}_{J}=\mathcal{T} \Psi^{\dagger}(L) \Psi(0)+\text { h.c. } .
$$

The above equation takes into account the possibility for a boson to tunnel through the barrier potential, and h.c. refers to the Hermitian conjugate corresponding to tunneling events in the opposite direction. In the hydrodynamic formulation for $\Psi^{\dagger}(x)$, the transfer of one boson is ensured by the operator $\exp (-i \varphi)$ where $\varphi=\phi(L)-\phi(0)$. By neglecting the density fluctuations in the field operator (5) we then recover the usual Josephson Hamiltonian

$$
\mathcal{H}_{J}=E_{J} \cos \varphi
$$

where $E_{J}=2 \rho_{0} \mathcal{T}$ is the Josephson energy of the junction.

Quantum fluctuations of the bosons in the ring on both sides of the barrier tend to smear the phase $\varphi$ and hence suppress the tunneling strength. Indeed, from the diagonal Hamiltonian of section $\amalg B$ the ring constitutes an oscillator bath for the junction with linear spectrum $\hbar \omega_{k_{j}}=\pi \hbar v_{S} j / L$; the resulting model is very similar to the one describing a superconducting Josephson junction coupled to a resistive environment [21]. Tunneling events thus induce excitations of the modes of the ring with energy between $\hbar \omega_{0}=\pi \hbar v_{S} / L$ and the high energy cutoff $\hbar \omega_{h}=\pi \hbar v_{S} / \alpha$.

When the Josephson energy is smaller than the lowest mode $\hbar \omega_{0}$, corresponding to small rings $L<L^{*} \sim$ $\hbar v_{S} / E_{J}$, the junction can be treated as a perturbation and every mode modifies $E_{J}$. The effective Josephson energy results from averaging $\mathcal{H}_{J}$, Eq. (28), with respect to the ground state of the unperturbed Hamiltonian (2): $E_{J}^{\mathrm{eff}}=\left\langle\mathcal{H}_{J}\right\rangle$ with $\left\langle\mathcal{H}_{J}\right\rangle / E_{J}=\mathcal{G}_{0}(L, \alpha) / \rho_{0}$. Then

$$
E_{J}^{\mathrm{eff}}=E_{J}\left(\frac{\pi \alpha}{2 L}\right)^{1 / K} \quad \text { for } L<L^{*} .
$$

The Josephson energy decreases with the power law $L^{-1 / K}$ of the one-body density for probe points at the edges of the ring. This case includes the limit of an infinitely high barrier, where $E_{J} \rightarrow 0, L^{*} \rightarrow \infty$, and which is illustrated in Fig. 2, bottom panel.

When $E_{J}$ is larger than $\hbar \omega_{0}$ (with $E_{J}<\hbar \omega_{h}$ ), only the modes with energies larger than $E_{J}$ contribute to the renormalization. Consequently, to obtain the effective Josephson energy we need to average over wavelengths between $\alpha$ and the characteristic length $\ell \equiv \pi \hbar v_{S} / E_{J}^{\text {eff }}$ : $E_{J}^{\mathrm{eff}}=\left\langle\left\langle\mathcal{H}_{J}\right\rangle\right\rangle$ with

$$
E_{J}^{\mathrm{eff}} / E_{J}=\mathcal{G}_{0}(L, \alpha) / \mathcal{G}_{0}(\ell, \alpha) \sim(\alpha / \ell)^{1 / K}
$$

The effective Josephson energy in this case is obtained by solving the above self-consistent equation with respect to $\ell$, with the result

$$
E_{J}^{\mathrm{eff}}=E_{J}\left(\frac{\alpha E_{J}}{\pi \hbar v_{S}}\right)^{1 /(K-1)} \quad \text { for } L>L^{*} .
$$

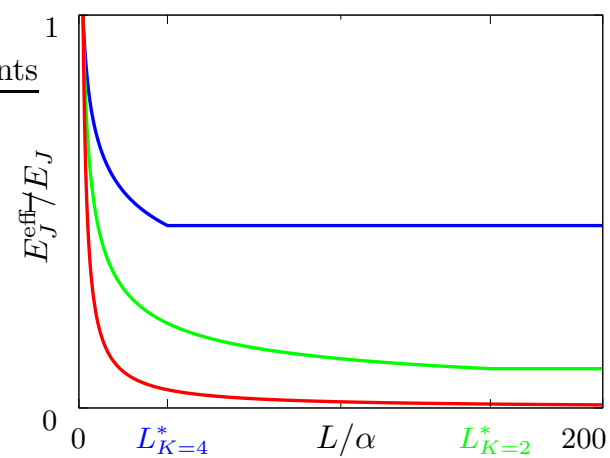

FIG. 6: (Color online) Renormalized Josephson energy as a function of the length of the ring. From top to bottom $K=$ 4, 2, 1 with $\hbar \omega_{h}=10 E_{J} . \quad E_{J}^{\text {eff }}$ decreases as $1 / L^{1 / K}$ and reaches a constant value at $L=L^{*}$.

In this case $E_{J}^{\mathrm{eff}}$ is independent of the ring circumference $L$. Our results (29) and (31) are summarized in Fig. 6. As a main conclusion, we find that quantum fluctuations dramatically reduce the tunnel amplitude with respect to its bare value entering the Hamiltonian (27), especially in the case $K=1$. Note however that the reduction saturates at a nonzero level for rings larger than the so-called healing length $L^{*}$. The continuity between the two regimes $L<L^{*}$ and $L>L^{*}$ defines the healing length of the ring $L^{*}=\pi \alpha / 2\left(\pi \hbar v_{S} / \alpha E_{J}\right)^{K /(K-1)}$.

As a final remark, we would like to mention that our approach is equivalent to the renormalization group formalism [22] or the self-consistent harmonic approximation [21].

\section{SUMMARY AND CONCLUDING REMARKS}

In summary, in this paper we have studied the equilibrium properties of a quasi-1D interacting Bose gas confined in a ring trap with a localized barrier. In the limit of infinite barrier we have studied the coherence, density profiles and density-density correlations of the gas using a Luttinger-liquid approach and the quantum average method. Our results recover and extend those previously known by the use of conformal field theory methods. As physical consequences of our analysis, we find that the one-body density matrix, when probed at various points with respect to the barrier position, is expected to display universal power law behaviors with different exponents which depend only on the Luttinger parameter $K$. We also find that our method permits to describe accurately the Friedel oscillations (due to the presence of the barrier) occurring in the particle density profile and in the density-density correlation function. Once the non-universal parameters entering the effective model are fixed by comparing the density profile to the exact one in the Tonks-Girardeau case $K=1$, we find that the Luttinger liquid model well agrees with the exact result for the density-density correlation function at 
a length scale which is at the boundary of the validity of the Luttinger-liquid model.

The analysis performed in the limit of infinite barrier is then use to study perturbatively the presence of a large, finite barrier. By taking into account the effect of quantum fluctuations we find that the effective Josephson energy (i.e. the tunnel amplitude across the barrier) is reduced with respect to its bare value, in a way which depends on the length of the ring with respect to a typical healing length, a maximal reduction occurring for long rings.

The effect of the renormalization of the tunnel am- plitude is expected to have strong consequences on the dynamical evolution of the ring-trapped Bose gas with a Josephson junction.

\section{Acknowledgments}

We acknowledge stimulating discussions with R. Citro, L.I. Glazman, J. Schmiedmayer. We thank IUF, CNRS and the MIDAS-STREP project for financial support.
[1] S. Gupta et al., Phys. Rev. Lett. 95, 143201 (2005); O. Morizot et al., Phys. Rev. A 74, 023617 (2006); C. Ryu et al., Phys. Rev. Lett. 99, 260401 (2007).

[2] V.N. Popov, Theor. Math. Phys. 11, 478 (1972); V.N. Popov, Functional integrals in Quantum Field Theory and Statistical Physics (Reidel, Dordrecht, 1983), Chap. 6.

[3] D.S. Petrov, G.V. Shlyapnikov, and J.T. Walraven, Phys. Rev. Lett. 85, 3745 (2000).

[4] M. Girardeau, J. Math. Phys. 1, 516 (1960); M.D. Girardeau, Phys. Rev. 139, B500 (1965).

[5] F. Cataliotti et al., Science 293, 843 (2001); M. Albiez et al., Phys. Rev. Lett. 95, 010402 (2005); Y. Shin et al., Phys. Rev. Lett. 95, 170402 (2005); S. Levy et al., Nature 449, 579 (2007); J. Sebby-Strabley et al. Phys. Rev. Lett. 98, 200405 (2007); G.-B. Jo et al., Phys. Rev. Lett. 98, 030407 (2007); S. Foelling et al., Nature 448, 1029 (2007); J. Esteve et al., arXiv:0810.0600.

[6] M.A. Cazalilla, J. Phys. B 37, S1 (2004).

[7] T. Giamarchi, Quantum Physics in One Dimension, Oxford University Press (2003).

[8] A. Lenard, Pacific J. Math. 42, 137 (1972).

[9] P.J. Forrester, N.E. Frankel, T.M. Garoni, and N.S. Witte1, Phys. Rev. A 67, 043607 (2003).

[10] F.D.M. Haldane, Phys. Rev. Lett. 47, 1840 (1981); J.
Phys. C 14, 2585 (1981).

[11] S. Ritter et al., Phys. Rev. Lett. 98, 090402 (2007).

[12] F.W.J. Hekking and L.I. Glazman, Phys. Rev. B 55, 6551 (1997).

[13] A. Minguzzi, P. Vignolo and M.P. Tosi, Physics Letters A 294, 222 (2002).

[14] M. Olshanii, V. Dunjko, Phys. Rev. Lett., 91, 090401 (2003); New J. Phys., 5, 98 (2003).

[15] A. De Martino, M. Thorwart, R. Egger, and R. Graham, Phys. Rev. Lett. 94, 060402 (2005).

[16] J. Friedel, N. Cimento Suppl. 7, 287 (1958).

[17] R. Egger, H. Grabert, in Quantum Transport in Semiconductor Submicron Structures edited by B. Kramer, NATO-ASI Series E (Kluwer, Dordrecht, 1996).

[18] M.D. Girardeau, A. Minguzzi, arXiv:0807.3366

[19] In absence of known results for the case of arbitrary $K$, we have adopted the same choice of parameters $\alpha$ and $\theta_{B}$ for any value of $K$.

[20] P. Vignolo, A. Minguzzi, and M.P. Tosi, Phys. Rev. A 64, 023421 (2001).

[21] G. Schön and A.D. Zaikin, Phys. Rep. 198, 237 (1990).

[22] C.L. Kane and M.P.A. Fisher, Phys. Rev. Lett. 68, 1220 (1992). 